\title{
Demonstration of tailored energy deposition in a laser proton accelerator
}

\author{
J. G. Zhu $\odot,{ }^{1,2, \star}$ M. J. Wu, ${ }^{1, \star}$ K. Zhu, ${ }^{1,2}$ Y. X. Geng, ${ }^{1,2}$ Q. Liao, ${ }^{1}$ D. Y. Li, ${ }^{1}$ T. Yang, ${ }^{1}$ \\ M. J. Easton $\odot,{ }^{1,2}$ C. C. $\mathrm{Li},{ }^{1}$ X. H. Xu ${ }^{1}$ Y. R. Shou, ${ }^{1}$ J. Q. Yu, ${ }^{1}$ Z. Gong, ${ }^{1}$ Y. Y. Zhao, ${ }^{1,2}$ \\ P. J. Wang $\odot,{ }^{1}$ D. H. Wang, ${ }^{1}$ L. Tao, ${ }^{1}$ C. E. Chen, ${ }^{1,2}$ W. J. Ma, ${ }^{1,2}$ H. Y. Lu, ${ }^{1,2}$ T. Tajima, ${ }^{3}$ \\ G. Mourou, ${ }^{4}$ C. Lin, ${ }^{1,2, *}$ and X. Q. Yan ${ }^{1,2, \uparrow}$ \\ ${ }^{1}$ State Key Laboratory of Nuclear Physics and Technology, and Key Laboratory of HEDP of the Ministry \\ of Education, CAPT, Peking University, Beijing, China, 100871 \\ ${ }^{2}$ Beijing Laser Acceleration Innovation Center, Beijing, China, 101407 \\ ${ }^{3}$ Department of Physics and Astronomy, UC Irvine, Irvine, California 92697, USA \\ ${ }^{4}$ DER-IZEST, Ecole Polytechnique, 91128 Palaiseau Cedex, France
}

(Received 20 March 2020; accepted 20 November 2020; published 28 December 2020)

\begin{abstract}
In order to implement radiotherapy based on a laser accelerator, it is necessary to precisely control the spatial distribution and energy spectrum of the proton beams to meet the requirements of the radiation dose distribution in the three-dimensional biological target. A compact laser plasma accelerator has been built at Peking University, which can reliably generate and transport MeV-energy protons with a specified energy onto the irradiation platform. In this paper, we discuss several technologies for the accurate control of a laser-accelerated proton beam with large divergence angle and broad energy spread, including the determination of the beam source position with micron accuracy, a tuning algorithm for the transport line which we refer to as "matching-image-point two-dimensional energy analysis" to realize accurate energy selection, and the control of beam distribution uniformity. In the prototype experiment with low energy protons and $0.5-\mathrm{Hz}$ irradiation rate, a tailored energy deposition is demonstrated, which shows the potential feasibility of future irradiation based on laser-accelerated proton beams.
\end{abstract}

DOI: 10.1103/PhysRevAccelBeams.23.121304

\section{INTRODUCTION}

Particle accelerators have made a huge impact on modern society. A host of different kinds of accelerators are in operation around the world for diverse applications, such as studying the collisions of quarks with each other in the field of high-energy physics, investigations of collisions of heavy nuclei, synchrotron light sources in the study of atomic structure and biology, and proton beam radiation therapy for cancer treatment. There are now more than 60 proton radiotherapy centers where thousands of patients with malignant tumors are treated each year. Traditional accelerators for proton therapy are large and expensive, so significant development is focused on producing more compact and cheaper accelerators [1]. High-intensity laser accelerators have been suggested as a potential alternative [2].

\footnotetext{
*Corresponding author. 1c0812@pku.edu.cn

Corresponding author. x.yan@pku.edu.cn

These authors are equally contributed to this work.

Published by the American Physical Society under the terms of the Creative Commons Attribution 4.0 International license. Further distribution of this work must maintain attribution to the author(s) and the published article's title, journal citation, and DOI.
}

When an ultrahigh-intensity laser is focused on a solid target, high-energy-density electrons are expelled from the target, establishing an acceleration field with a gradient exceeding TV/m, capable of accelerating ions to multi$\mathrm{MeV}$ energies within a distance of tens of microns [3-5]. The advantage of using plasma as acceleration medium is that it can provide orders of magnitude higher gradients, which has the potential to shrink down the size compared to traditional ion accelerators. The most widely employed laser ion acceleration mechanisms are target normal sheath acceleration (TNSA) [6-8], radiation pressure acceleration (RPA) [9-13] and break-out afterburner acceleration (BOA) [14]. With a petawatt (PW) laser system, the maximum proton energy accelerated in the TNSA regime is $85 \mathrm{MeV}$ with micron thickness targets [15], and is nearly $100 \mathrm{MeV}$ in the RPA and BOA regimes with nanometerthin foils $[16,17]$. Despite the relatively low energy transfer efficiency, TNSA is considered the most robust and stable mechanism, where the ions (especially protons) from the surface contamination layer are accelerated by the charge separation sheath field. However, due to the rapid diffusion of the electron layer, Coulomb explosion and multiple instabilities, TNSA ion beams are characterized by an exponentially decaying energy spectrum and a large divergence angle, which are quite different from the output of conventional accelerators [18]. One of the comments from 
2018's Nobel Physics Committee was that "there are many remaining challenges before laser accelerators can be used for medical applications" [19].

In recent research, various beam line designs have been proposed to collect, deliver and utilize this "imperfect" proton beam [20-23]. For instance, GSI Helmholtz Center built the LIGHT beam line and experimentally demonstrated tight focusing of a nearly $10 \mathrm{MeV}$ proton beam with improved homogeneity [24,25]. The ELIMED beam line, which is designed to transport $60 \mathrm{MeV}$ proton beams for therapeutic purposes will be commissioned soon [26]. BELLA at LBNL has proposed a compact superconducting gantry design [27]. Masood proposed a compact gantry design with $360^{\circ}$ rotation capability for laser-driven proton acceleration for radiotherapy [28]. Spectral and spatial shaping of a laser-produced ion beam has been researched for radiation-biology applications [29,30]. A compact laser plasma accelerator (CLAPA) with a fully functional beam line composed of electromagnets has also been built at Peking University [31], and proton beams with 3 to $9 \mathrm{MeV}$ energy and $1 \%$ energy spread were transported to the irradiation platform with precise parameter control.

To obtain a stable beam distribution which accurately meets the requirements of the irradiation parameters, every step of beam control is critical, including collimation, focusing, energy analyzing and homogenization. For the special application of cancer therapy, a higher-level requirement, spectrum shaping, should be met if the wide energy spectrum is tailored to produce a spread-out Bragg peak (SOBP). That means that the original exponentially decaying spectrum of laser-accelerated protons should be shaped to a specified ascending energy spectrum according to the requirement of the SOBP. Hence accurate energy analysis of beam with a large divergence angle and energy spread is an important prerequisite.

In practical applications, stable operation of laser accelerators is still a challenge. For example, misalignment of the proton source could cause inaccurate energy selection and deviation of beam spot position at the irradiation point, which should be strictly controlled in cancer therapy. In this paper, we show several technologies for the accurate control of a laser-accelerated proton beam with large divergence angle and broad energy spread through a beam line composed of electromagnets. First, we show how to precisely determine the beam source position with micron accuracy to achieve proton beams with high stability and repeatability. Then, we have studied the transverse emittance growth in focusing and bending elements due to the chromatic effects, and their influences on energy analyzing and particle distribution uniformity. We also demonstrate the improvement of proton beam distribution uniformity through the optics of the beam line. Based on these technologies, we were able to produce tailored energy deposition with a three-dimensional radially symmetric dose distribution from a TW laser accelerator operated at
$0.5 \mathrm{~Hz}$. Although the demonstrated deposition depth is only a hundred microns with $\mathrm{MeV}$ protons, it still proves the potential of laser accelerators. We propose the use of matching-image-point two-dimensional energy analysis to realize accurate analyzing for particles with a large divergence angle and energy spread, with which it will be feasible to shape the typically broad, exponential-like energy spectrum to meet the requirements for cancer therapy.

\section{DESIGN OF THE BEAM LINE}

The experiments were carried out on the CLAPA platform at Peking University. The laser contrast was $10^{-10}$ at 40 ps before the main pulse using an a cross-polarized wave (XPW) filter, and no plasma mirror system was used. In the experiment, a p-polarized laser pulse with $1.3 \mathrm{~J}$ energy (43 TW) and $30 \mathrm{fs}$ duration was focused onto a $2.5-\mu \mathrm{m}-$ thick aluminum target using an $\mathrm{f} / 3.5$ off-axis parabola at an incident angle of $30^{\circ}$ to the target normal direction. The spot diameter (FWHM) was $5 \mu \mathrm{m}$ with $25 \%$ of the total energy, corresponding to an intensity of $5.5 \times 10^{19} \mathrm{~W} / \mathrm{cm}^{2}$ on the target. A high-magnification imaging system was used to ensure accurate coupling between the laser focal spot and each target, with a spatial resolution of $0.1 \mu \mathrm{m}$. CLAPA generates a high-current proton beam with an exponentially decaying energy distribution and a divergence angle of about $\pm 10^{\circ}$ [32]. The typical energy

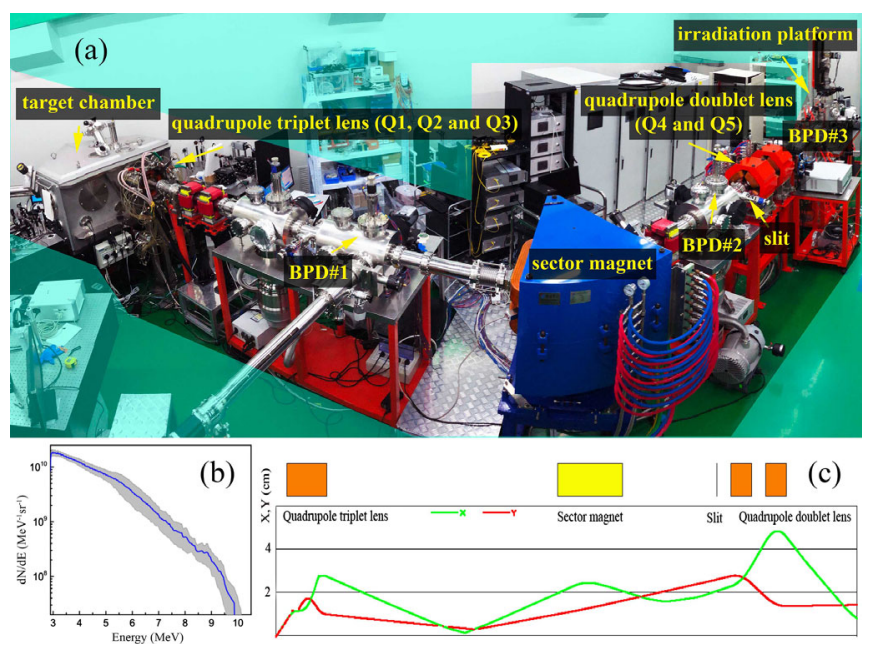

FIG. 1. CLAPA beam line: (a) Panoramic photograph of the beam line. (b) Measurement of the proton spectrum with a Thomson spectrometer placed $140 \mathrm{~mm}$ behind the target based on a $2.5 \mu \mathrm{m}$ aluminum foil [32]. The solid line is averaged over ten shots and the gray area is the rms deviation. During the beam line experiment, this Thomson spectrometer was replaced by the quadrupole triplet lens. (c) Simulated envelope evolution of the proton beam for a tailored energy distribution with a central energy of $4.62 \mathrm{MeV}$ and energy spread of $\pm 3 \%$, using the program TRACK. The radius of the initial beam spot is set to $5 \mu \mathrm{m}$ and the acceptance angle is $\pm 50 \mathrm{mrad}$. 
TABLE I. Length of each element along the beam line.

\begin{tabular}{lc}
\hline \hline Element & Length $(\mathrm{cm})$ \\
\hline Target & 0 \\
Drift & 19 \\
Q1 & 10 \\
Drift & 5.9 \\
Q2 & 20 \\
Drift & 5.2 \\
Q3 & 10 \\
Drift & 356.5 \\
B1 & 51.1 \\
Drift & 177.5 \\
Q4 & 30 \\
Drift & 20 \\
Q5 & 30 \\
Drift & 102 \\
RCF (radiochromic films) & \\
\hline \hline
\end{tabular}

spectrum detected with a magnetic energy spectrometer is shown in Fig. 1(b).

\section{A. Collection and determination of beam source position}

Figure 1(a) shows the panoramic picture and the detailed setup of the CLAPA beam line. The length of each element and the parameters of magnets are shown in Tables I and II. The first part of the beam transport system is a quadrupole triplet lens (composed of Q1, Q2 and Q3) inserted into the vacuum chamber, which collects and gathers protons from the laser plasma acceleration within a divergence angle of $\pm 50 \mathrm{mrad}$ [33]. The quadrupole triplet lens is demonstrated experimentally to be an object-to-image point transport system at the central energy, with a certain amplification factor. If Q3 is turned off, Q1 and Q2 can act as a quadrupole doublet lens and then the amplification factor will vary. For instance, the amplification factors $(F)$ in the $y$ (vertical) direction can be calculated to be $-6.94\left(F_{1}\right)$ and $-2.96\left(F_{2}\right)$ respectively with Q3 switched on and off, when the image point is positioned at BPD\#1 (beam profile detector \#1, using a scintillator). In the $x$ (horizontal) direction, the corresponding magnification factors are -3.61 and -23.46 .

When the beam source (the laser irradiation point) has a displacement from the beam center line (namely the center

TABLE II. Parameters of quadrupole and sector magnets.

\begin{tabular}{lccc}
\hline \hline Element & Aperture & Maximum magnetic field & Current \\
\hline Q1 & $3 \mathrm{~cm}$ & $5 \mathrm{KGs} / \mathrm{cm}$ & $300 \mathrm{~A}$ \\
Q2 & $6.4 \mathrm{~cm}$ & $2.5 \mathrm{KGs} / \mathrm{cm}$ & $540 \mathrm{~A}$ \\
Q3 & $6.4 \mathrm{~cm}$ & $2.5 \mathrm{KGs} / \mathrm{cm}$ & $540 \mathrm{~A}$ \\
B1 & $7 \mathrm{~cm}($ gap) & $1.5 \mathrm{~T}$ & $850 \mathrm{~A}$ \\
Q4 & $11 \mathrm{~cm}$ & $0.727 \mathrm{KGs} / \mathrm{cm}$ & $220 \mathrm{~A}$ \\
Q5 & $11 \mathrm{~cm}$ & $0.727 \mathrm{KGs} / \mathrm{cm}$ & $220 \mathrm{~A}$ \\
\hline \hline
\end{tabular}

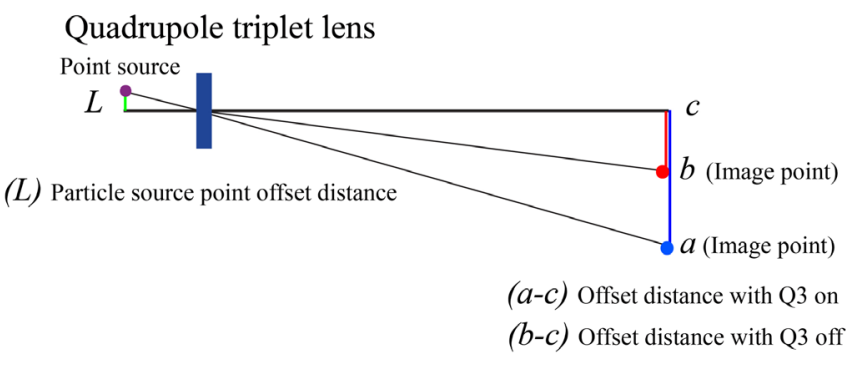

FIG. 2. Schematic diagram of focusing with the quadrupole triplet lens when the beam source has a displacement from the beam center line in the $y$ direction. Triplet lens (Q3 on) and doublet lens (Q3 off) have different amplification factors.

axis of the triplet lens), the beam spot at the image point will have a certain displacement according to the amplification factor in the $x$ (horizontal) or $y$ direction. Assuming the beam source displaces $L$ from the center line of the triplet lens, then the beam positions at $a$ and $b$ with Q3 switched on and off can be measured at BPD\#1, as shown in Fig. 2. Then the actual beam line center $c$ at BPD\#1 can be determined, which is on the center line of the triplet lens. The beam source displacement $L$ can be calculated by this relation:

$$
L=\frac{a-c}{F_{1}}=\frac{b-c}{F_{2}} .
$$

Then the beam source can be moved to the beam center line by adjusting the target control system.

For example, by moving the beam source up and down $0.66 \mathrm{~mm}$ in the $y$ direction, the beam spot positions at BPD\#1 show certain variation, according to the amplification factors, and we can obtain the beam line center $c$ in these three conditions, as shown in Fig. 3(a). The crossing point in Fig. 3(a) indicates the real required beam source position, which means that we can move $0.53 \mathrm{~mm}$ in the $y$ direction to locate the beam line center [to the crossing point in Fig. 3(a)]. As the displacement of the beam spot from the beam line center reflects that of the beam source, fluctuations of the beam source positions can be measured by the fluctuations of the beam spot positions at BPD\#1. Figures 3(b) and 3(c) show fluctuations of beam spot positions of seven shots in the $x$ and $y$ directions. Fluctuations of the beam source position can be calculated to be within $\pm 27 \mu \mathrm{m}$. The experiments proved that the beam source positions in the $x$ and $y$ directions can be measured and adjusted independently.

\section{B. Energy analysis}

After passing the quadrupole triplet lens, the beam is focused at the focal spot in the $x$ direction, which is the object point of a 45-degree sector magnet in an object-toimage point transport system. After energy analysis in the 
(a)

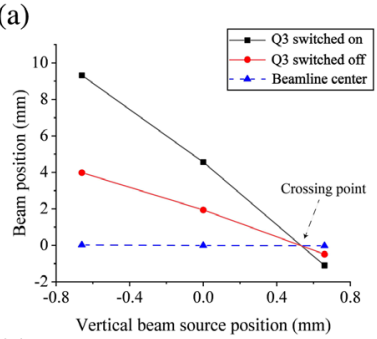

(c)

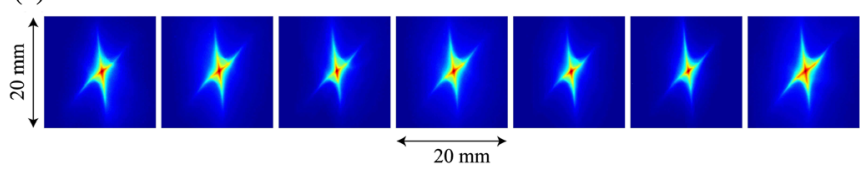

FIG. 3. Determining the displacement of the beam source and fluctuations of the beam spot. (a) By moving the beam source up and down $0.66 \mathrm{~mm}$ in the $y$ direction, beam spot positions (black and red dots) at BPD\#1 show remarkable variations, and beam line center $c$ (blue dots) can be obtained. The coordinate origin of the $x$ axis is the initial position of the beam source. The coordinate origin of the $y$ axis is determined by the average beam line centers $c$ at BPD\#1. The crossing point indicates the real beam source position $(0.53 \mathrm{~mm}$ on the $x$ axis). (b) Fluctuations of beam spot center measured on BPD\#1. Positions of the beam center are converted from pixel values. (c) Seven beam spots measured on BPD\#1.

$x$ (horizontal) direction, protons with the same energy and different angular divergence converge to the same image point; protons with different energies are dispersed along the $x$ axis.

The horizontal emittance will increase due to chromatic aberration when passing through the sector magnet. We calculated the transverse emittance $\varepsilon_{x, y}$ growth with the transformation matrix in this quadrupole triplet lens under different conditions, when the $5 \mathrm{MeV}$ proton beam was focused onto BPD\#1. $\varepsilon_{x, y}$ growth declines rapidly with the increase of initial beam radius. Figure 4(a) shows that $\varepsilon_{x, y}$ growth is approximately linear with divergence angle, with a fixed beam source radius of $5 \mu \mathrm{m}$ and energy spread of $\pm 1 \%$. Figure 4(b) shows $\varepsilon_{x, y}$ growth is approximately linear with energy spread with a fixed initial divergence angle of $\pm 10 \mathrm{mrad}$. The dots in Fig. 4(b) are the $\varepsilon_{x, y}$ growth for the beams with $\pm 50 \mathrm{mrad}$ divergence angle and $\pm 3 \%$ energy spread, which were used to accumulate the spread-out Bragg peak in the following experiment. Hence transverse emittance growth is inevitable when focusing laser-driven beams with a small initial beam size (several micrometers) and large divergence angle and energy spread.

After the energy analysis through the sector magnet, the emittance in the horizontal $\left(x, x^{\prime}\right)$ phase space increases further. The black dots in Fig. 4(d) show the normalized root mean square (rms) emittance $\mathcal{E}_{x, \text { rms }}$ of 11 monoenergetic proton beams with certain energy differences from $5 \mathrm{MeV}(0 \%, \pm 1 \%, \pm 2 \%, \pm 3 \%, \pm 4 \%, \pm 5 \%)$ and with
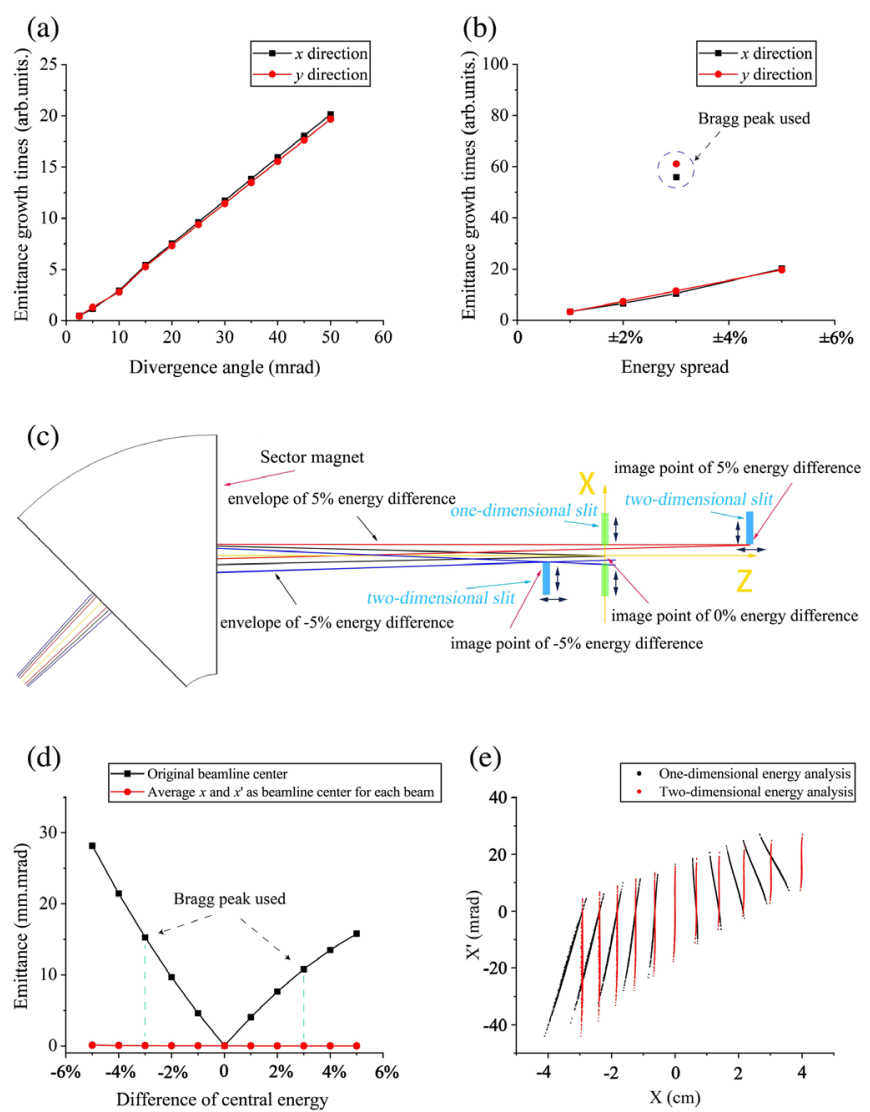

FIG. 4. Emittance growth under different conditions, and emittance with new beam line center. (a) Transverse emittance growth is approximately linear with divergence angle. The initial beam radius is $5 \mu \mathrm{m}$ and the energy spread is $\pm 1 \%$. (b) Transverse emittance growth is approximately linear with energy spread. The initial beam radius is $5 \mu \mathrm{m}$ and the initial divergence angle is $\pm 10 \mathrm{mrad}$. The initial divergence angle for dots circled in blue is $\pm 50 \mathrm{mrad}$, which is used in the production of the tailored energy distribution. (c) Schematic comparing selection with onedimensional slit and two-dimensional slits. Black lines represent the envelope of protons at the central energy. Red and blue lines represent the envelopes of protons with $+5 \%$ and $-5 \%$ energy difference respectively. These results are calculated using the program TRACK. (d) Emittance growth with and without adjusting the beam line center. Black dots show the normalized rms emittances of proton beams with different energy spreads. Red dots show new horizontal normalized rms emittance of each beam with a new beam line center determined by both the average position and the divergence angle. (e) Distributions of eleven monoenergetic proton beams in the horizontal $\left(x, x^{\prime}\right)$ phase space. The rightmost beam is the beam with $5 \%$ energy difference. Black dots show the results with one-dimensional energy analysis and red dots show the results with matching-image-point twodimensional energy analysis.

\pm 50 mrad initial divergence angle. The initial distribution is a water-bag distribution with an initial beam source radius of $5 \mu \mathrm{m}$. This emittance is given by $\mathcal{E}_{x, \text { rms }}{ }^{2}=$ $\beta^{2} \gamma^{2}\left(\left\langle x^{2}\right\rangle\left\langle x^{\prime 2}\right\rangle-\left\langle x x^{\prime}\right\rangle^{2}\right)$. Transport is simulated by the program TRACK. The quadrupole triplet lens is set as a 
hard edge, and the sector magnet has a fringe field. The calculation shows that the rms emittance of the beam with $5 \%$ energy difference increases by more than 4600 times, from an initial 0.0034 to $15.81 \pi \mathrm{mm}$ mrad.

Normally, after the sector magnet, a slit in the $x$ direction is used to select the proton energy. However, beams with different energies have different transverse and longitudinal positions for the image point after being focused by the quadrupoles and analyzed by the sector magnet. We propose to use the matching-image-point two-dimensional energy analysis, where energy selection is performed at the image point of each beam with a certain energy by translating the slit both along the $x$ and $z$ axes to track the focal points at each energy, as shown in Fig. 4(c). Distributions of the eleven monoenergetic proton beams in the horizontal $\left(x, x^{\prime}\right)$ phase space are shown in Fig. 4(e), after one-dimensional energy analysis (black dots) or twodimensional energy analysis (red dots). This shows that energy selection can be more accurate with the twodimensional energy analysis as each beam gets converged in the $x$ direction.

The "actual" horizontal emittance relating to the energy-analyzing ability for each beam can be calculated according to its beam line center, which is determined by the average position and divergence angle of each beam. This new horizontal normalized rms emittance for each beam increases much less, as shown by the red dots in Fig. 4(d). For example, with the new beam line center the emittance of the beam with 5\% energy difference and $\pm 50 \mathrm{mrad}$ initial divergence angle increases by 7 times, from an initial 0.0034 to $0.027 \pi \mathrm{mm}$ mrad. The emittance growth of 7 times is mainly attributed to the deviation from linear focusing of quadrupoles. If the quadrupoles have a fringe field, the growth times will be larger. It shows that with appropriate selection of beam line parameters, in spite of the broad energy spectra and large divergence in laserdriven beams, small emittance can be preserved, and good quality beams can be analyzed and prepared for different applications. As the SOBP requires an ascending spectrum, the exponentially decaying energy spectrum should be shaped when the energy spread is large. Hence twodimensional energy analysis makes energy deposition tailoring feasible for laser-driven beams.

We tested the energy-analyzing ability of the sector magnet using the foil cutoff method (illustrated in Fig. 5). By adjusting the image point of the sector magnet onto BPD\#3 using a microchannel plate $(\mathrm{MCP})$, protons with a continuous energy distribution were separated along the $x$ axis on the MCP. We attached a 70- $\mu \mathrm{m}$-thick aluminum foil in front of the MCP (corresponding to a proton cutoff energy of $2.75 \mathrm{MeV}$ ), so that protons with different energies were selectively shielded. The discrimination of energies is determined by the transportation efficiencies of the beam line and the transmission efficiencies through the aluminum foil, as shown in Fig. 5(a). We measured three
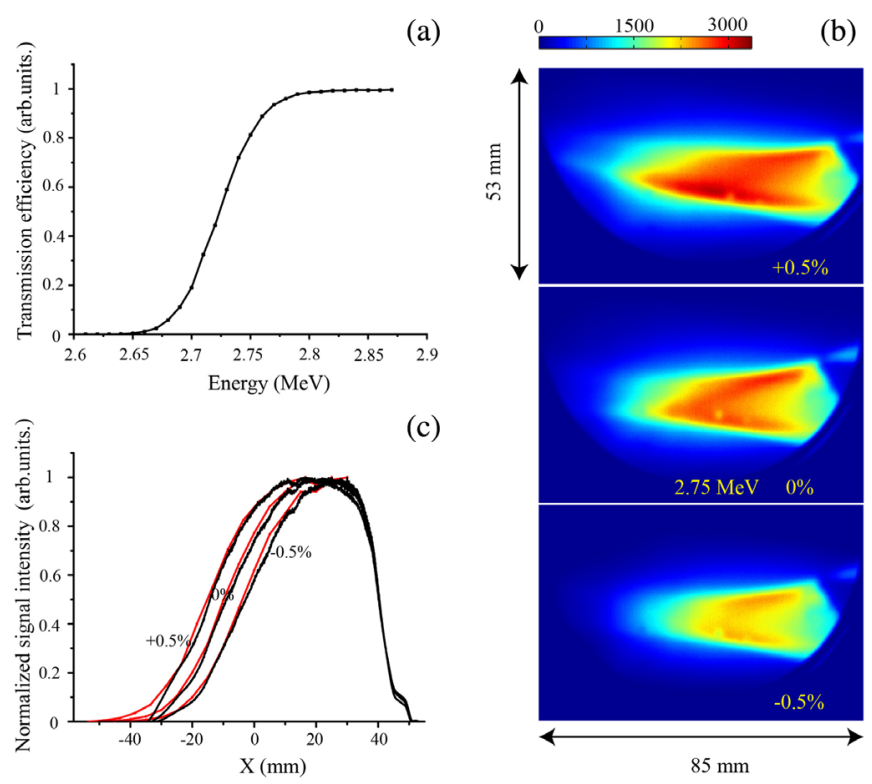

FIG. 5. Energy resolution testing using the foil cutoff method. (a) Transmission efficiencies of protons with different energies through a $70-\mu \mathrm{m}$-thick aluminum foil. The quadrupole doublet magnet was turned off and the image point of the sector magnet was adjusted onto the MCP. (b) Proton beam profiles on the MCP with central energies differing from $2.75 \mathrm{MeV}$ by $0.5 \%, 0 \%$ and $-0.5 \%$ respectively from top to bottom. (c) Normalized signal intensity along the $x$ direction for the three energy conditions. From left to right, the differences of central energies are $0.5 \%, 0 \%$ and $-0.5 \%$ from $2.75 \mathrm{MeV}$, respectively. The black curves are the experimental data and the red curves are theoretical data.

bunches of proton beams at the MCP, with central energies differing from $2.75 \mathrm{MeV}$ by $0.5 \%, 0 \%$, and $-0.5 \%$ respectively, as shown in Fig. 5(b). Signal intensity along the $y$ direction is summed up for the three conditions to produce a position-energy-transmission curve. The experimental data (black curves) are normalized to compare with theoretical calculation results (red curves), as shown in Fig. 5(c). The detected transmission efficiencies at the same $x$ coordinate have remarkable and predictable differences under the three conditions, which confirm the accuracy of energy and the energy-selecting ability of the system. This shows that the CLAPA beam line has the ability to control proton energies with accuracy better than $0.5 \%$.

When the image point of the central energy is adjusted onto the slit, by setting the width of the slit to $14 \mathrm{~mm}$, protons with $\pm 1 \%$ energy spread can be selected and focused onto the irradiation platform by a quadrupole doublet lens positioned after the sector magnet, as shown in Fig. 1.

\section{Controlling of particle distribution uniformity}

Uniformity of the particle distribution at the irradiation point is a necessary prerequisite for accurate dose control. Our particle beam optics is carefully designed to obtain 
(a)

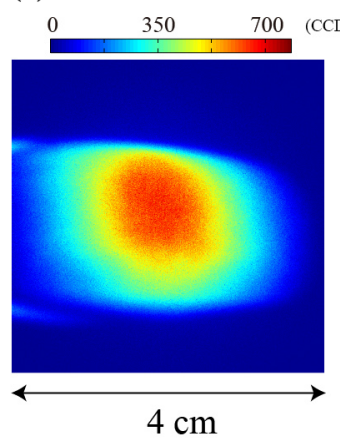

(c)

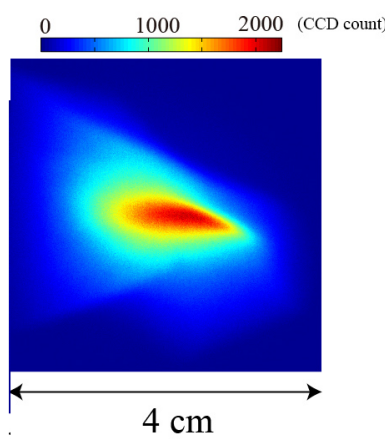

(b)

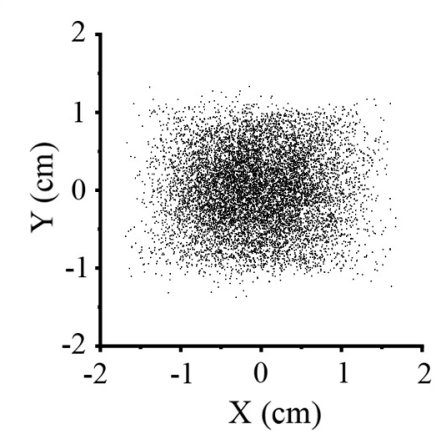

(d)

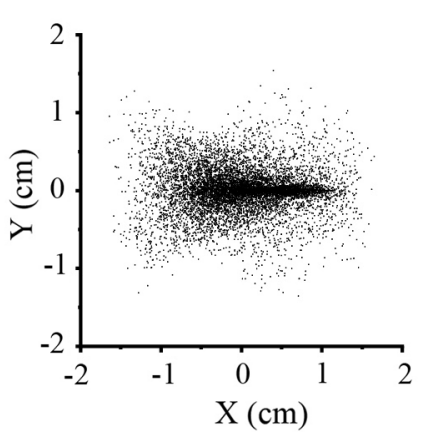

FIG. 6. Control of the beam profile at the irradiation point. Experimental (a) and simulated (b) demonstrations of radially symmetric distributions of $5 \mathrm{MeV}$ proton beams with $\pm 1 \%$ energy spread on BPD\#3 (using a MCP). Experimental (c) and simulated (d) demonstrations of malformed beam spots with improper focal length of the triplet lens in the y direction.

radially symmetric proton distributions: the focal length of the triplet lens in the $y$ direction can be freely adjusted to manipulate the proton distribution evolution in the $y$ direction; and the doublet lens regulates the beam profile and size, and determines the final distribution [34]. Figures 6(a) and 6(b) show the experimental and simulated demonstrations of a radially symmetric distribution of $5 \mathrm{MeV}$ proton beams with $\pm 1 \%$ energy spread. In the case of transverse emittance growth [as the black dots shown in Fig. 4(d)], radially symmetric beams can be realized from the laser accelerator. If there is a mismatch of the focal length of the triplet lens in the $y$ direction, the beam spot is malformed, as shown in Figs. 6(c) and 6(d), which can be caused by the change of the magnetic field gradients of Q2 and Q3 from -1.102 and $1.286 \mathrm{kG} / \mathrm{cm}$ to -1.084 and $1.276 \mathrm{kG} / \mathrm{cm}$.

\section{PRODUCTION OF SOBP}

The narrow Bragg peak of monoenergetic protons can be broadened to the spread-out Bragg peak (SOBP) by combining separate shots at different energies, which is one of the key technologies of radiotherapy and requires high-precision beam control of energy, energy spread,

energy spectrum, beam source position, beam spot size and uniformity in a laser accelerator. We tested this ability by accumulating 203 shots of laser-driven protons with central energies being $3.45 \mathrm{MeV}$ (two shots), $3.66 \mathrm{MeV}$ (four shots), $3.88 \mathrm{MeV}$ (six shots), $4.11 \mathrm{MeV}$ (15 shots), 4.36 MeV (30 shots), 4.62 MeV (146 shots) and energy spread of $\pm 3 \%$, then the total energy range is from 3.35 to 4.76 MeV. We used the beam line to select the proton beams from the initial exponentially decaying energy spectra [the dashed black line in Fig. 7(b)], and accumulated them into an ascending energy spectrum [the red curve in Fig. 7(b)], so as to obtain the tailored energy deposition required for SOPB at the irradiation platform. The slit size is set as $\pm 20 \mathrm{~mm}$ to obtain $\pm 3 \%$ energy spread. As the energy spread of $\pm 3 \%$ is not large, the exponentially decaying spectrum of each shot is utilized directly without energy shaping. These 203 shots were continuously accumulated at $0.5 \mathrm{~Hz}$.

(a)

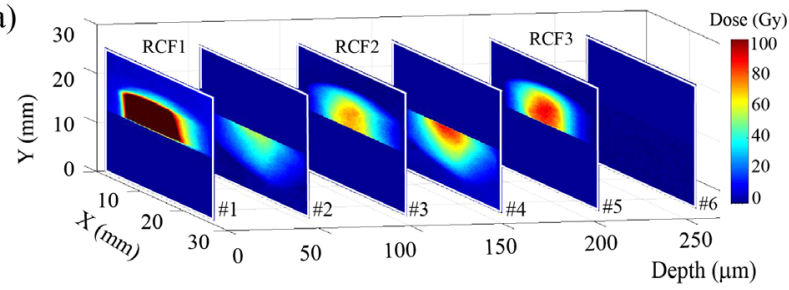

(b)

(c)
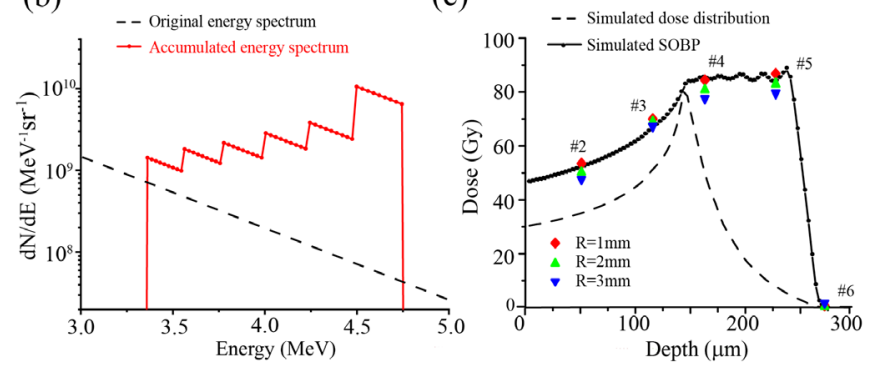

FIG. 7. Production of a spread-out Bragg peak with accumulated proton shots at different energies. (a) Dose distributions at different depths recorded on the RCF stack. The depth shift of the Bragg peak due to the aluminum foil is converted to an equivalent depth for the RCF film. Emulsion of the RCF layer is $8 \mu \mathrm{m}$, and the thickness of RCF is measured as $111 \mu \mathrm{m}$. (b) The black dashed curve is the exponentially decaying energy spectrum which is retrieved from the RCF records. The red curve is the total spectrum of proton beams accumulated to form the SOBP. (c) Comparison of experimental results with simulated depth profile. The black curve is the calculated accumulated dose distributions of all 203 shots of protons, as simulated using SRIM. The red, green, and blue dots represent the average doses within a 1,2, and $3 \mathrm{~mm}$ radius of the beam center, respectively, at the different depths obtained in the experiment. The black dashed curve represents the expected dose profile if we use the exponentially decaying energy spectrum directly. The dose coordinate of the black dashed curve is magnified for ease of comparison, while the shape of the curve is not changed. 
A stack of three layers of HD-V2 radiochromic films (RCFs) was used to record the three-dimensional dose distributions. An aluminum foil $30 \mu \mathrm{m}$ thick was put in front of the lower half of the first RCF, so that the corresponding Bragg peak energy in each RCF sensitive layer (the lower part) was shifted, as shown in Table III. We can see that the dose distributions at different depths of energy deposition are nearly radially symmetric, as shown in Fig. 7(a). Using a $\pm 3 \%$ energy spread increases utilization of the proton beams and maintains a radially symmetric proton distribution. The contamination layer on the target contains carbon, hydrogen and oxygen, so that carbon and oxygen ions with different charge state were also accelerated together with protons. Although the beam line was set to transport a proton beam with aimed central energy of $E$, heavy ions with certain charge to mass ratio $q / A$ and energy of $q^{2} \times E / A$ had the same beam rigidity and were inevitably transmitted with the same transverse envelope with protons in the beam line composed of only magnetic field components. The penetration depth of heavy ions is much smaller than that of protons at the same energy, hence they can be easily removed by a thin foil. In Fig. 7(a), the upper half of the first RCF acts as a shielding for these heavy ions, which caused excessive dosage.

Based on our previous calibrations [35], the energy loss in each RCF layer was shown in Table III. The number of protons in the $4.62 \mathrm{MeV}$ beam with a $\pm 3 \%$ energy spread was calculated as $1.6 \times 10^{7}$ per pulse at the irradiation platform. The average radiation dose rate is approximately $17 \mathrm{~Gy} / \mathrm{min}$. In the experiment, the receiving angle of the quadrupole triplet lens was $\pm 50 \mathrm{mrad}$, corresponding to a proton collection efficiency of $30 \%$ [33]. For the collected protons with the aimed energy and $\pm 3 \%$ energy spread, the transmission efficiency of the beam line was about $88 \%$.

Figure 7(c) shows the calculated dose distribution (black curve) at different depths and the experimental average doses within $1 \mathrm{~mm}$ (red diamond), $2 \mathrm{~mm}$ (green triangle) and $3 \mathrm{~mm}$ (blue triangle) radius of the beam spots. The results show a good fit to the expected depth profile, demonstrating that it is possible to produce an SOBP with a laser accelerator. The black dashed curve in Fig. 7(c) shows the expected dose curve if we used the exponentially decaying spectrum directly, for comparison. If we used

TABLE III. Corresponding Bragg peak energy, depth and energy loss in each RCF.

\begin{tabular}{lccc}
\hline \hline & & Depth $/ \mu \mathrm{m}$ & \\
& Energy/MeV & $(\mathrm{Al}+\mathrm{RCF})$ & Energy loss/J \\
\hline 1\#RCF1 upper & 0.5 & 4 & $2.1 \times 10^{-4}$ \\
2\#RCF1 lower & 1.8 & $30+4$ & $3.9 \times 10^{-5}$ \\
3\#RCF2 upper & 3.0 & 115 & $5.4 \times 10^{-5}$ \\
4\#RCF2 lower & 3.6 & $30+115$ & $6.6 \times 10^{-5}$ \\
5\#RCF3 upper & 4.4 & 226 & $5.8 \times 10^{-5}$ \\
6\#RCF3 lower & 4.9 & $30+226$ & 0 \\
\hline \hline
\end{tabular}

the same number of shots for each central energy, then the total energy spectrum would simply be proportional to the section of the original exponentially decaying spectrum [the black dashed in Fig. 7(b)] from 3.35 to $4.76 \mathrm{MeV}$. Depositing this untailored energy distribution at the irradiation point would produce a dose distribution as shown by the black dashed curve in Fig. 7(c). Hence tailoring the numbers of shots for different central energies changes the final dose depth profile, and enables an initially exponentially decaying spectrum to satisfy the requirements of the SOBP.

\section{SUMMARY}

In summary, the CLAPA experimental results show a laser plasma accelerator integrated with an image-relaying beam line that can reliably deliver protons with beam qualities suitable for many applications. Transverse emittance growth of laser-driven beams is inevitable in focusing and energy analysis, but the "actual" final transverse emittance is still small. With two-dimensional energy analysis, the exponentially decaying spectrum of laserdriven beams with large divergence angle and energy spread can be tailored according to the requirements of the SOBP. We produced a tailored energy deposition with a three-dimensional radially symmetric dose distribution. The ion optics proposed in this paper can realize the homogenization of a point source with a large divergence angle and wide energy spread, including, but not limited to, laser-driven beams. Although the magnet technologies used for this beam line are not novel, the ion optic design used in producing such a tailored energy deposition is new and crucial to this work. Hopefully, with the development of high-repetition-rate petawatt laser technology [36] and image-relaying ion optics, the laser accelerator will be a truly viable alternative, suitable for many applications.

\section{ACKNOWLEDGMENTS}

The research has been supported by the NSFC (11975037, 11921006, 11775010, 61631001), and the National Grand Instrument Project (No. 2019YFF01014400 and No. 2019YFF01014404).

[1] T. R. Bortfeld and J.S. Loeffler, Three ways to make proton therapy affordable, Nature (London) 549, 451 (2017).

[2] T. Tajima, Compact Laser Proton Accelerator beyond $100 \mathrm{MeV}$ for Medicine (LLNL, Livermore, 1999).

[3] A. Macchi, M. Borghesi, and M. Passoni, Ion acceleration by superintense laser-plasma interaction, Rev. Mod. Phys. 85, 751 (2013).

[4] C. M. Ma, I. Veltchev, E. Fourkal, J. S. Li, W. Luo, J. Fan, T. Lin, and A. Pollack, Development of a laser-driven 
proton accelerator for cancer therapy, Laser Phys. 16, 639 (2006).

[5] H. Daido, M. Nishiuchi, and A. S. Pirozhkov, Review of laser-driven ion source and their applications, Rep. Prog. Phys. 75, 056401 (2012).

[6] B. M. Hegelich, B. J. Albright, J. Cobble, K. Flippo, S. Letzring, M. Paffett, H. Ruhl, J. Schreiber, R. K. Schulze, and J.C. Fernández, Laser acceleration of quasi-monoenergetic $\mathrm{MeV}$ ion beams, Nature (London) 439, 441 (2006).

[7] R. A. Snavely, M. H. Key, S. P. Hatchett, T. E. Cowan, M. Roth, T. W. Phillips, M. A. Stoyer, E. A. Henry, T. C. Sangster, M. S. Singh, S. C. Wilks, A. MacKinnon, A. Offenberger, D. M. Pennington, K. Yasuike, A. B. Langdon, B. F. Lasinski, J. Johnson, M. D. Perry, and E. M. Campbell, Intense High-Energy Proton Beams from Petawatt-Laser Irradiation of Solids, Phys. Rev. Lett. 85, 2945 (2000).

[8] J. Fuchs, P. Antici, E. d'Humières, E. Lefebvre, M. Borghesi, E. Brambrink, C. A. Cecchetti, M. Kaluza, V. Malka, M. Manclossi, S. Meyroneinc, P. Mora, J. Schreiber, T. Toncian, H. Pépin, and P. Audebert, Laser-driven proton scaling laws and new paths towards energy increase, Nat. Phys. 2, 48 (2006).

[9] T. Esirkepov, M. Borghesi, S. V. Bulanov, G. Mourou, and T. Tajima, Highly Efficient Relativistic-Ion Generation in the Laser-Piston Regime, Phys. Rev. Lett. 92, 175003 (2004).

[10] X. Q. Yan, C. Lin, Z. M. Sheng, Z. Y. Guo, B. C. Liu, Y. R. Lu, J. X. Fang, and J. E. Chen, Generating High-Current Monoenergetic Proton Beams by a Circularly Polarized Laser Pulse in the Phase-Stable Acceleration Regime, Phys. Rev. Lett. 100, 135003 (2008).

[11] S. Kar, K. F. Kakolee, B. Qiao, A. Macchi, M. Cerchez, D. Doria, M. Geissler, P. McKenna, D. Neely, J. Osterholz, R. Prasad, K. Quinn, B. Ramakrishna, G. Sarri, O. Willi, X. Y. Yuan, M. Zepf, and M. Borghesi, Ion Acceleration in Multispecies Targets Driven by Intense Laser Radiation Pressure, Phys. Rev. Lett. 109, 185006 (2012).

[12] S. M. Weng, Z. M. Sheng, M. Murakami, M. Chen, M. Liu, H. C. Wang, T. Yuan, and J. Zhang, Optimization of hole-boring radiation pressure acceleration of ion beams for fusion ignition, Matter Radiat. Extremes 3, 28 (2018).

[13] V. K. Tripathi, T. C. Liu, and X. Shao, Laser radiation pressure proton acceleration in gaseous target, Matter Radiat. Extremes 2, 256 (2017).

[14] L. Yin, B. J. Albright, K. J. Bowers, D. Jung, J. C. Fernández, and B. M. Hegelich, Three-Dimensional Dynamics of Breakout Afterburner Ion Acceleration Using High-Contrast Short-Pulse Laser and Nanoscale Targets, Phys. Rev. Lett. 107, 045003 (2011).

[15] F. Wagner, O. Deppert, C. Brabetz, P. Fiala, A. Kleinschmidt, P. Poth, V. A. Schanz, A. Tebartz, B. Zielbauer, M. Roth, T. Stöhlker, and V. Bagnoud, Maximum Proton Energy Above $85 \mathrm{MeV}$ from the Relativistic Interaction of Laser Pulses with Micrometer Thick $\mathrm{CH}_{2}$ Targets, Phys. Rev. Lett. 116, 205002 (2016).

[16] I. J. Kim, K. H. Pae, I. W. Choi, C. L. Lee, H. T. Kim, H. Singhal, J. H. Sung, S. K. Lee, H. W. Lee, P. V. Nickles,
T. M. Jeong, C. M. Kim, and C. H. Nam, Radiation pressure acceleration of protons to $93 \mathrm{MeV}$ with circularly polarized petawatt laser pulses, Phys. Plasmas 23, 070701 (2016).

[17] A. Higginson, R. J. Gray, M. King, R. J. Dance, S. D. R. Williamson, N. M. H. Butler, R. Wilson, R. Capdessus, C. Armstrong, J. S. Green, S. J. Hawkes, P. Martin, W. Q. Wei, S. R. Mirfayzi, X. H. Yuan, S. Kar, M. Borghesi, R. J. Clarke, D. Neely, and P. McKenna, Near-100 MeV protons via a laser-driven transparency-enhanced hybrid acceleration scheme, Nat. Commun. 9, 724 (2018).

[18] U. Linz and J. Alonso, Laser-driven ion accelerators for tumor therapy revised, Phys. Rev. Accel. Beams 19, 124802 (2016).

[19] High-Intensity, Generation of Groundbreaking Inventions in Laser Physics (2018), http://galaxy.uci.agh.edu.pl/ rozlow/fizyka/Nagroda\%20Nobla\%20z\%20Fizyki\% 202018/advanced-physicsprize2018.pdf.

[20] T. Toncian, M. Borghesi, J. Fuchs, E. d'Humières, P. Antici, P. Audebert, E. Brambrink, C. A. Cecchetti, A. Pipahl, L. Romagnani, and O. Willi, Ultrafast laser-driven microlens to focus and energy-select mega-electron volt protons, Science 312, 410 (2006).

[21] M. Schollmeier et al., Controlled Transport and Focusing of Laser-Accelerated Protons with Miniature Magnetic Devices, Phys. Rev. Lett. 101, 055004 (2008).

[22] K. Harres, I. Alber, A. Tauschwitz, V. Bagnoud, H. Daido, M. Günther, F. Nürnberg, A. Otten, M. Schollmeier, J. Schütrumpf, M. Tampo, and M. Roth, Beam collimation and transport of quasineutral laser-accelerated protons by a solenoid field, Phys. Plasmas 17, 023107 (2010).

[23] I. Hofmann, Performance of solenoids versus quadrupoles in focusing and energy selection of laser accelerated protons, Phys. Rev. ST Accel. Beams 16, 041302 (2013).

[24] D. Jahn, D. Schumacher, C. Brabetz, J. Ding, S. Weih, F. Kroll, F. E. Brack, U. Schramm, A. Blažević, and M. Roth, First application studies at the laser-driven LIGHT beam line: Improving proton beam homogeneity and imaging of a solid target, Nucl. Instrum. Methods Phys. Res., Sect. A 909, 173 (2018).

[25] D. Jahn, D. Schumacher, C. Brabetz, F. Kroll, F. E. Brack, J. Ding, R. Leonhardt, I. Semmler, A. Blažević, U. Schramm, and M. Roth, Focusing of multi-MeV, subnanosecond proton bunches from a laser-driven source, Phys. Rev. Accel. Beams 22, 011301 (2019).

[26] F. Romano, F. Schillaci, G. A. P. Cirrone, G. Cuttone, V. Scuderi, L. Allegra, A. Amato, A. Amico, G. Candiano, G. De Luca, G. Gallo, S. Giordanengo, L. FanolaGuarachi, G. Korn, G. Larosa, R. Leanza, R. Manna, V. Marchese, and A. Vignati, The ELIMED transport and dosimetry beam line for laser-driven ion beams, Nucl. Instrum. Methods Phys. Res., Sect. A 829, 153 (2016).

[27] Q. Ji, S. Bulanov, S. Steinke, T. Schenkel, E. H. Esarey, and W. P. Leemans, Design of a compact ion beam transport system for the BELLA ion accelerator, in Proceedings of the 7th International Particle Accelerator Conference (IPAC'16), Busan, Korea, 2016 (JACOW, Geneva, Switzerland, 2016), pp. 3391-3393. 
[28] U. Masood, M. Bussmann, M. Bussmann, T. E. Cowan, W. Enghardt, L. Karsch, F. Kroll, U. Schramm, and J. Pawelke, A compact solution for ion beam therapy with laser accelerated protons, Appl. Phys. B 117, 41 (2014).

[29] F. E. Brack, F. Kroll, L. Gaus, C. Bernert, E. Beyreuther, T. E. Cowan, L. Karsch, S. Kraft, L. A. Kunz-Schughart, E. Lessmann, J. Metzkes-Ng, L. Obst-Huebl, J. Pawelke, M. Rehwald, H. Schlenvoigt, U. Schramm, M. Sobiella, E. R. Szabó, T. Ziegler, and K. Zeil, Spectral and spatial shaping of laser-driven proton beams using a pulsed highfield magnet beam line, Sci. Rep. 10, 9118 (2020).

[30] L. Pommarel, B. Vauzour, F. Mégnin-Chanet, E. Bayart, O. Delmas, F. Goudjil, C. Nauraye, V. Letellier, F. Pouzoulet, F. Schillaci, F. Romano, V. Scuderi, G. A. P. Cirrone, E. Deutsch, A. Flacco, and V. Malka, Spectral and spatial shaping of a laser-produced ion beam for radiation-biology experiments, Phys. Rev. Accel. Beams 20, 032801 (2017).

[31] J. G. Zhu et al., Experimental demonstration of a laser proton accelerator with accurate beam control through image-relaying transport, Phys. Rev. Accel. Beams 22, 061302 (2019).
[32] Y.X. Geng et al., Generating proton beams exceeding $10 \mathrm{MeV}$ using high contrast $60 \mathrm{TW}$ laser, Chin. Phys. Lett. 35, 092901 (2018).

[33] M. J. Wu, J. G. Zhu, D. Y. Li, T. Yang, Q. Liao, Y. X. Geng, X. H. Xu, C. C. Li, Y. R. Shou, Y. Y. Zhao, Y. R. Lu, H. Y. Lu, W. J. Ma, C. Lin, K. Zhu, and X. Q. Yan, Collection and focusing of laser accelerated proton beam by an electromagnetic quadrupole triplet lens, Nucl. Instrum. Methods Phys. Res., Sect. A 955, 163249 (2020).

[34] J. G. Zhu, K. Zhu, L. Tao, X. H. Xu, C. Lin, W. J. Ma, H. Y. Lu, Y. Y. Zhao, Y. R. Lu, C. E. Chen, and X. Q. Yan, Distribution uniformity of laser-accelerated proton beams, Chin. Phys. C 41, 097001 (2017).

[35] X. H. Xu, Q. Liao, M. J. Wu, Y. X. Geng, D. Y. Li, J. G. Zhu, C. C. Li, R. H. Hu, Y. R. Shou, Y. H. Chen, H. Y. Lu, W. J. Ma, Y. Y. Zhao, K. Zhu, C. Lin, and X. Q. Yan, Detection and analysis of laser driven proton beams by calibrated Gafchromic HD-V2 and MD-V3 radiochromic films, Rev. Sci. Instrum. 90, 033306 (2019).

[36] G. Mourou, B. Bill, T. Toshiki, and L. Jens, The future is fiber accelerators, Nat. Photonics 7, 258 (2013). 